la revue La revue pour l'histoire du CNRS

POUR L'HISTOIRE DU CNRS $13 \mid 2005$

Regards sur des laboratoires en sciences humaines et sociales

\title{
La signature scientifique. Une sociologie pragmatique de l'attribution
}

David Pontille. CNRS ÉDITIONS, Paris, 2004

\section{Muriel Le Roux}

\section{OpenEdition \\ Journals}

Édition électronique

URL : https://journals.openedition.org/histoire-cnrs/442

DOI : $10.4000 /$ histoire-cnrs.442

ISSN : 1955-2408

Éditeur

CNRS Éditions

\section{Édition imprimée}

Date de publication : 3 novembre 2005

ISBN : 978-2-271-06349-6

ISSN : 1298-9800

Référence électronique

Muriel Le Roux, « La signature scientifique. Une sociologie pragmatique de l'attribution », La revue pour I'histoire du CNRS [En ligne], 13 | 2005, mis en ligne le 07 mars 2006, consulté le 20 mai 2021. URL: http://journals.openedition.org/histoire-cnrs/442 ; DOI : https://doi.org/10.4000/histoire-cnrs.442

Ce document a été généré automatiquement le 20 mai 2021.

Comité pour l'histoire du CNRS 


\section{La signature scientifique. Une sociologie pragmatique de l'attribution}

David Pontille. CNRS ÉDITIONS, Paris, 2004

\section{Muriel Le Roux}

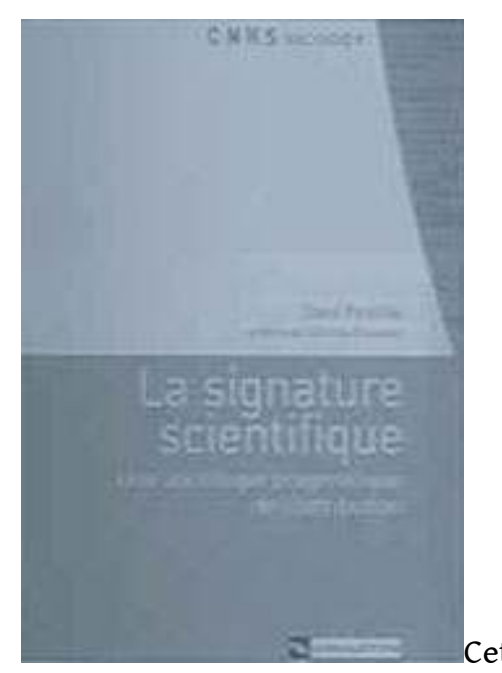

Cet ouvrage de sociologie nous décrit ce que recouvre le fait de signer un texte pour un chercheur. Ce geste simple, qui accompagne la recherche et clôt la publication, n'est pas seulement cet acte « ordinaire » qui signale à l'autre l'identité de l'auteur et engage sa responsabilité sur le contenu du message. Alors que chacun sait qu'en matière juridique la seule signature engage de facto la personne, nombre d'actes passés au cours d'une vie sont clos par la mention « lu et approuvé » ce qui n'ajoute rien en droit mais rassure et rend plus solennel l'acte de signer.

Puisqu'il en est ainsi des documents les plus simples que nous signions, qu'en est-il pour une publication scientifique? David Pontille a mené une enquête selon les critères de sa discipline en observant et en interrogeant des biologistes, des juristes et des sociologues. 
2 D'un strict point de vue scientifique, il aurait été sans doute plus opportun de ne garder la référence aux pratiques sociologiques que par comparaison et choisir une autre discipline des sciences humaines, le champ aurait été plus large et l'analyse plus distanciée. Par ailleurs, on aurait aimé trouver une conclusion plus étoffée, celle de l'ouvrage opère comme si l'auteur avait totalement absorbé les pratiques de "l'autorialité " décrite abondamment sous toutes ses formes notamment pour la biologie. Le sociologue s'efface totalement derrière son objet, ce qui est louable, mais alors que la bibliographie (abondante sur le sujet) est présente tout au long de l'ouvrage, à la fin, on ne peut pas ne pas se demander quelle était la question de départ de l'auteur. Ce petit défaut excepté, ce livre intéressant décrit tous les enjeux qui se nouent autour de la présence d'un ou de plusieurs noms au bas d'un article ainsi que les règles qui en régissent le classement. La biologie est une discipline où le travail collectif constitue la règle, la sociologie occupant une place médiane tandis que le droit est une discipline où l'exercice de rédaction est individuel.

3 L'auteur présente les revues, leurs classements, les indices de citation, rappelant souvent avec intérêt la genèse des processus qui sont devenus les éléments de référence structurant la carrière des chercheurs.

4 La signature symbolise toute l'histoire de la discipline depuis la pratique individuelle jusqu'au mode de fonctionnement collectif. Il ressort que cette histoire est aussi celle du mode d'organisation de la communauté scientifique.

5 Si l'ouvrage restitue fidèlement les observations (les annexes donnent à voir ce que le chercheur a amassé et comment il a travaillé), la tendance à la généralisation, qui est sans doute la conséquence d'un jeu de plume plus qu'une volonté réelle, masque une réalité qui est bien plus complexe qu'il n'y paraît, pour le plus grand malheur des responsables de politique scientifique partout dans le monde.

6 Car imaginer que les règles de la biochimie s'appliquent à toutes les sciences dures serait inexact. Ainsi dans certains laboratoires de physique, certains directeurs refusent la règle de l'ascenseur (un nom, un service avec ou sans participation à la recherche en échange de... sur une publication) et en instaurent d'autres comme : « ne signent un papier que ceux qui ont tout d'abord participer de façon décisive à la recherche et ensuite parmi eux, ceux qui sont capables d'expliquer et défendre le papier devant les pairs réunis en séminaire, colloque ou congrès ». Cela a le mérite de clarifier les enjeux. En chimie, il existe encore d'autres pratiques car les liens avec les entreprises étant fréquents, le secret prévaut parfois excluant toute publication avant que les découvertes brevetées ne soient en phase de développement. L'évaluation des chercheurs emprunte alors d'autres chemins. Que dire de l'histoire, discipline où certains chercheurs mettent leurs compétences scientifiques et techniques au service de la communauté sans jamais être cités ni même remerciés. Alors imaginer un article signé collectivement relève presque de la déraison et dans tous les cas «ne sert par la carrière ».

7 Ce que souligne avant tout ce livre est qu'un travail de recherche prend du temps, qu'il n'est complet que lorsque la reconnaissance de ce travail, à savoir l'évaluation, est faite correctement. Pour cela les articles et dossiers scientifiques doivent être lus et les rapports en rendant compte doivent mettre en évidence la pertinence de la recherche. Mais cette activité est extrêmement chronophage et épuisante car elle n'entre pas officiellement dans la somme de travail que le chercheur a à fournir. 
Qui osera prétendre que tout cela est fait dans le plus pur respect des règles de la République des lettres en France alors que précisément ce travail de lecture, de compte-rendu n'est jamais reconnu comme inhérent au métier de chercheur, qu'il n'entre pas dans l'évaluation du dossier desdits évaluateurs? Pourtant, il s'agit d'un principe fondamental du fonctionnement de la recherche garant de son indépendance.

Alors que l'on vante souvent l'intégrité des procédures américaines, une enquête de l'université de Minneapolis montre qu'un tiers des chercheurs des National Institutes of Health, publiée dans Nature (9 juin 2005), ont reconnu avoir soit modifié des résultats obtenus pour préserver ou obtenir un budget, soit plagié des collègues ou encore utilisé leurs travaux sans les citer. L'enquête conclue que ces non-respects de l'éthique et de la déontologie menacent l'intégrité de la science. Ce n'est que la partie émergée de l'iceberg, car en réalité c'est la course contre le temps qui menace l'intégrité de la science en pesant sur les chercheurs. Aller vite, publier vite, sans vérifier les expériences ou les sources, conduisent à ces manquements qui sont autant de dérapages. Ils mettent en péril la recherche publique et/ou académique. Ces pratiques n'existent pas en milieu industriel sous ces formes-là. Le rapport temps/rentabilité est posé d'entrée de jeux comme un mode de fonctionnement. Les règles sont connues de tous.

Clarifier les modalités d'évaluation, donner du temps aux chercheurs, définir des règles claires, partagées et acceptées où le rapport entre le coût, l'investissement et la rentabilité espérée par le contribuable sont les préalables nécessaires pour soutenir la qualité d'une activité déterminante pour nos économies fondées sur le savoir. Ce livre a ouvert la boîte noire, aux décideurs des politiques scientifiques d'assumer avec les politiciens leurs responsabilités car il est certain que des mesures courageuses s'imposent.

\section{AUTEUR}

\section{MURIEL LE ROUX}

Chargée de recherche au CNRS (IHMC) 